\title{
Cryptanalysis of RadioGatún
}

\author{
Thomas Fuhr ${ }^{1}$ and Thomas Peyrin ${ }^{2}$ \\ 1 DCSSI Labs \\ thomas.fuhr@sgdn.gouv.fr \\ 2 Ingenico \\ thomas.peyrin@ingenico.com
}

\begin{abstract}
In this paper we study the security of the RadioGatun family of hash functions, and more precisely the collision resistance of this proposal. We show that it is possible to find differential paths with acceptable probability of success. Then, by using the freedom degrees available from the incoming message words, we provide a significant improvement over the best previously known cryptanalysis. As a proof of concept, we provide a colliding pair of messages for RadioGatún with 2-bit words. We finally argue that, under some light assumption, our technique is very likely to provide the first collision attack on RadioGatún.
\end{abstract}

Keywords: hash functions, RadioGatún, cryptanalysis.

\section{Introduction}

A cryptographic hash function is a very important tool in cryptography, used in many applications such as digital signatures, authentication schemes or message integrity. Informally, a cryptographic hash function $H$ is a function from $\{0,1\}^{*}$, the set of all finite length bit strings, to $\{0,1\}^{n}$ where $n$ is the fixed size of the hash value. Moreover, a cryptographic hash function must satisfy the properties of preimage resistance, 2nd-preimage resistance and collision resistance [27]:

- collision resistance: finding a pair $x \neq x^{\prime} \in\{0,1\}^{*}$ such that $H(x)=$ $H\left(x^{\prime}\right)$ should require $2^{n / 2}$ hash computations.

- 2nd preimage resistance: for a given $x \in\{0,1\}^{*}$, finding a $x^{\prime} \neq x$ such that $H(x)=H\left(x^{\prime}\right)$ should require $2^{n}$ hash computations.

- preimage resistance: for a given $y \in\{0,1\}^{n}$, finding a $x \in\{0,1\}^{*}$ such that $H(x)=y$ should require $2^{n}$ hash computations.

Generally, hash functions are built upon a compression function and a domain extension algorithm. A compression function $h$, usually built from scratch, should have the same security requirements as a hash function but takes fixed length inputs instead. Wang et al. 32, 33, 34, 35] recently showed that most standardized compression functions (e.g. MD5 or SHA-1) are not collision resistant. Then, a domain extension method allows the hash function to handle arbitrary length 
inputs by defining an (often iterative) algorithm using the compression function as a black box. The pioneering work of Merkle and Damgård [15, 28] provided to designers an easy way in order to turn collision resistant compression functions onto collision resistant hash functions. Even if preserving collision resistance, it has been recently shown that this iterative process presents flaws [16, 19, 20, 21. and new algorithms [1, 2, 7, 25, 26] with better security properties have been proposed.

Most hash functions instantiating the Merkle-Damgård construction use a block-cipher based compression function. Some more recent hash proposals are based on construction principles which are closely related to stream ciphers. For example we can cite Grindahl [24] or RadioGatún [4]. The underlying idea of stream-oriented functions is to first absorb $m$-bit message blocks into a big internal state of size $c+m$ using a simple round function, and then squeeze the hash output words out. As the internal state is larger than the output of the hash function, the cryptanalytic techniques against the iterative constructions can not be transposed to the case of stream-oriented functions. In 2007, Bertoni et al. published a new hash construction mode, namely the sponge functions [6]. At Eurocrypt 2008, the same authors [5] published a proof of security for their construction : when assuming that the internal function $F$ is a random permutation or a random transformation, then the sponge construction is indifferentiable from a random oracle up to $2^{c / 2}$ operations.

However, even though the same authors designed RadioGatún and defined the sponge construction, RadioGatún does not completely fulfill the sponge definition. For evident performance reasons, the internal function $F$ of RadioGatún is not a very strong permutation and this might lead to correlations between some input and output words. This threat is avoided by applying blank rounds (rounds without message incorporation) just after adding the last padded message word. More recently, some NIST SHA-3 candidates are using permutation-based modes as well, for example SHABAL [10], or sponge functions, for example Keccak [3].

Regarding the Grindahl family of hash functions, apart from potential slide attacks [18], it has been shown [23, 29] that it can not be considered as collision resistant. However, RadioGatún remains yet unharmed by the preliminary cryptanalysis 22]. The designers of RadioGatún claimed that for an instance manipulating $w$-bit words, one can output as much as $19 \times w$ bits and get a collision resistant hash function. That is, no collision attack should exist which requires less than $2^{9,5 \times w}$ hash computations. The designers also stated [4] that the best collision attack they could find (apart from generic birthday paradox ones) requires $2^{46 \times w}$ hash computations. A first cryptanalysis result by Bouillaguet and Fouque [8] using algebraic technique showed that one can find collisions for RadioGatún with $2^{24,5 \times w}$ hash computations. Finally, Khovratovich [22] described an attack using $2^{18 \times w}$ hash computations and memory, that can find collisions with the restriction that the IV must chosen by the attacker (semifree-start collisions).

Our Contributions. In this paper, we provide an improved cryptanalysis of RadioGatún regarding collision search. Namely, using an improved computer-aided 
backtracking search and symmetric differences, we provide a technique that can find a collision with $2^{11 \times w}$ hash computations and negligible memory. As a proof of concept, we also present a colliding pair of messages for the case $w=2$. Finally, we argue that this technique has a good chance to lead to the first collision attack on RadioGatún (the computation cost for setting up a complete collision attack is below the ideal bound claimed by the designers, but still unreachable for nowadays computers).

Outline. The paper is organized as follows. First, in Section 2, we describe the hash function proposal RadioGatún. Then, in Section 3, we introduce the concepts of symmetric differences and control words, that will be our two mains tools in order to cryptanalyze the scheme. In Section 4, we explain our differential path generation phase and in Section 5 we present our overall collision attack. Finally, we draw the conclusion in last section.

\section{Description of RadioGatún}

RadioGatún is a hash function using the design approach and correcting the problems of Panama [14], StepRightUp [13] or Subterranean [11, 13].

RadioGatún maintains an internal state of 58 words of $w$ bits each, divided in two parts and simply initialized by imposing the zero value to all the words. The first part of the state, the mill, is composed of 19 words and the second part, the belt, can be represented by a matrix of 3 rows and 13 columns of words. We denote by $M_{i}^{k}$ the $i$-th word of the mill state before application of the $k$-th iteration (with $0 \leq i \leq 18$ ) and $B_{i, j}^{k}$ represents the word located at column $i$ and row $j$ of the belt state before application of iteration $k$ (with $0 \leq i \leq 12$ and $0 \leq j \leq 2$ ).

The message to hash is first padded and then divided into blocks of 3 words of $w$ bits each that will update the internal state iteratively. We denote by $m_{i}^{k}$ the $i$-th word of the message block $m^{k}$ (with $0 \leq i \leq 2$ ). Namely, for iteration $k$, the message block $m^{k}$ is firstly incorporated into the internal state and then a permutation $P$ is applied on it. The incorporation process at iteration $k$ is defined by :

$$
\begin{array}{lll}
B_{0,0}^{k}=B_{0,0}^{k} \oplus m_{0}^{k} & B_{0,1}^{k}=B_{0,1}^{k} \oplus m_{1}^{k} & B_{0,2}^{k}=B_{0,2}^{k} \oplus m_{2}^{k} \\
M_{16}^{k}=M_{16}^{k} \oplus m_{0}^{k} & M_{17}^{k}=M_{17}^{k} \oplus m_{1}^{k} & M_{18}^{k}=M_{18}^{k} \oplus m_{2}^{k}
\end{array}
$$

where $\oplus$ denotes the bitwise exclusive or operation.

After having processed all the message blocks, the internal state is finally updated with $N_{b r}$ blank rounds (simply the application of the permutation $P$, without incorporating any message block). Eventually, the hash output value is generated by successively applying $P$ and then outputting $M_{1}^{k}$ and $M_{2}^{k}$ as many time as required by the hash output size. 


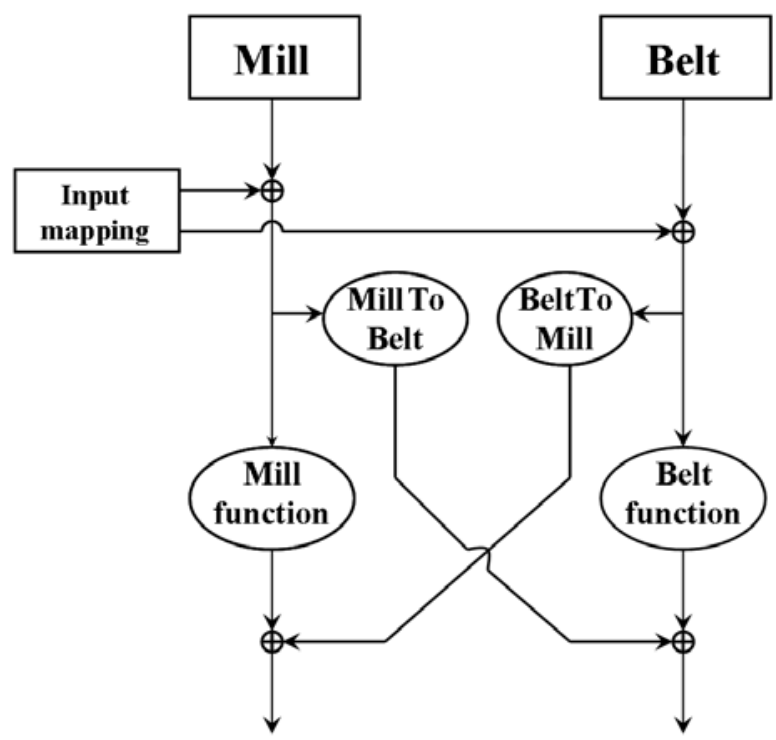

Fig. 1. The permutation $P$ in RadioGatún

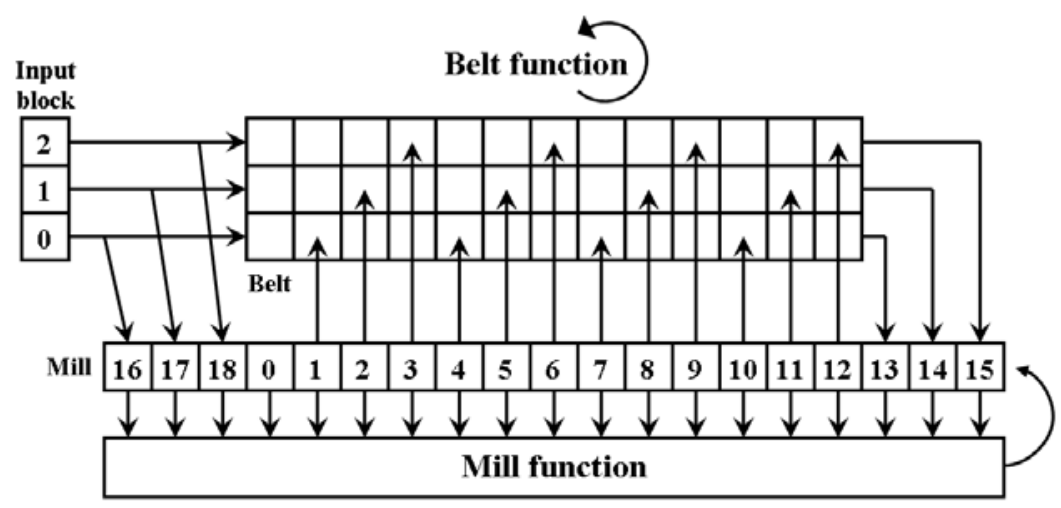

Fig. 2. The permutation $P$ in RadioGatún

The permutation $P$ can be divided into four parts. First, the Belt function is applied, then the MillToBelt function, the Mill function and eventually the BeltToMill function. This is depicted in Figures 1 and 2.

The Belt function simply consists of a row-wise rotation of the belt part of the state. That is, for $0 \leq i \leq 12$ and $0 \leq j \leq 2$ :

$$
B_{i, j}^{\prime}=B_{i+1 \bmod 13, j} .
$$




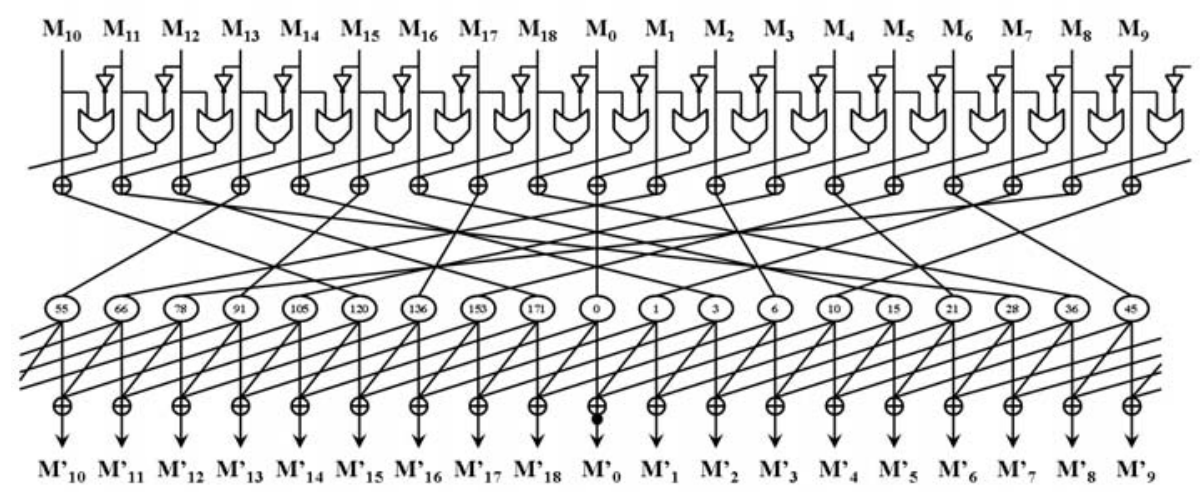

Fig. 3. The Mill function in RadioGatún

The MillToBelt function allows the mill part of the state to influence the belt one. For $0 \leq i \leq 11$, we have:

$$
B_{i+1, i \bmod 3}^{\prime}=B_{i+1, i \bmod 3} \oplus M_{i+1} .
$$

The Mill function is the most complex phase of the permutation $P$ and it updates the mill part of the state (see Figure 3). In the following, all indexes should be taken modulo 19. First, a nonlinear transformation is applied on all the words. For $0 \leq i \leq 18$ :

$$
M_{i}^{\prime}=M_{i} \oplus \overline{\overline{M_{i+1}}} \wedge M_{i+2}
$$

where $\bar{X}$ denotes the bitwise negation of $X$ and $\wedge$ represents the bitwise and operation. Then, a diffusion phase inside the words is used. For $0 \leq i \leq 18$ :

$$
M_{i}^{\prime}=M_{7 \times i} \ggg(i \times(i+1) / 2)
$$

where $X \ggg(y)$ denotes the rotation of $X$ on the right over $y$ positions. Then, a diffusion phase among all the words is applied. For $0 \leq i \leq 18$ :

$$
M_{i}^{\prime}=M_{i} \oplus M_{i+1} \oplus M_{i+4}
$$

Finally, an asymmetry is created by simply setting $M_{0}=M_{0} \oplus 1$.

The BeltToMill function allows the belt part of the state to influence the mill one. For $0 \leq i \leq 2$, we have :

$$
M_{i+13}^{\prime}=M_{i+13} \oplus B_{12, i}
$$

The RadioGatún security claims. Although RadioGatún has some common features with the sponge functions, the security proof of the sponge construction does not apply for this proposal. In their original paper [4], the authors claim that RadioGatún can output as much as 19 words and remain a secure hash function. Thus, it should not be possible for an attacker to find a collision attack running in less than $2^{9,5 \times w}$ hash computations. 


\section{Symmetric Differences and Control Words}

\subsection{Symmetric Differences}

The first cryptanalysis tool we will use are symmetric differences. This technique has first been described in [30]. It was mentioned as a potential threat for RadioGatún in [4]. More precisely, a symmetric difference is an intra-word exclusive or difference that is part of a stable subspace of all the possible differences on a $w$-bit word. For example, in the following we will use the two difference values $0^{w}$ and $1^{w}$ (where the exponentiation by $x$ denotes the concatenation of $x$ identical strings), namely either a zero difference or either a difference on every bit of the word.

Considering those symmetric differences will allow us to simplify the overall scheme. Regarding the intra-word rotations during the Mill function, a $0^{w}$ or a $1^{w}$ difference will obviously remain unmodified. Moreover, the result of an exclusive or operation between two symmetric differences will naturally be a symmetric difference itself:

$$
0^{w} \oplus 0^{w}=0^{w} \quad 0^{w} \oplus 1^{w}=1^{w} \quad 1^{w} \oplus 0^{w}=1^{w} \quad 1^{w} \oplus 1^{w}=0^{w}
$$

The nonlinear part of the Mill function is more tricky. We can write:

$$
\overline{\bar{a} \wedge b}=a \vee \bar{b} .
$$

The output of this transformation will remain a symmetric difference with a certain probability of success, given in Table 1 .

Table 1. Differential transitions for symmetric differences during the nonlinear part of the Mill function of RadioGatún. $\Delta_{a}$ and $\Delta_{b}$ denote the difference applied on $a$ and $b$ respectively, and $\Delta_{a \vee \bar{b}}$ the difference expected on the output of $a \vee \bar{b}$. The last column gives the corresponding conditions on the values of $a$ and $b$ in order to validate the differential transition. By $a=b$ (respectively $a \neq b$ ) we mean that all the bits of $a$ and $b$ are equal (respectively different), i.e. $a \oplus b=0^{w}$ (respectively $a \oplus b=1^{w}$ ).

\begin{tabular}{|c|c|c|c|c|}
\hline$\Delta_{a}$ & $\Delta_{b}$ & $\Delta_{a \vee \bar{b}}$ & Probability & Condition \\
\hline \hline $0^{w}$ & $0^{w}$ & $0^{w}$ & 1 & \\
\hline $0^{w}$ & $1^{w}$ & $0^{w}$ & $2^{-w}$ & $a=1^{w}$ \\
\hline $0^{w}$ & $1^{w}$ & $1^{w}$ & $2^{-w}$ & $a=0^{w}$ \\
\hline $1^{w}$ & $0^{w}$ & $0^{w}$ & $2^{-w}$ & $b=0^{w}$ \\
\hline $1^{w}$ & $0^{w}$ & $1^{w}$ & $2^{-w}$ & $b=1^{w}$ \\
\hline $1^{w}$ & $1^{w}$ & $0^{w}$ & $2^{-w}$ & $a=b$ \\
\hline $1^{w}$ & $1^{w}$ & $1^{w}$ & $2^{-w}$ & $a \neq b$ \\
\hline
\end{tabular}

Due to the use of symmetric differences, the scheme to analyze can now be simplified : we can concentrate our efforts on a $w=1$ version of RadioGatún, 
for which the intra-word rotations can be discarded. However, when building a differential path, for each differential transition during the nonlinear part of the Mill function, we will have to take the corresponding probability from Table 1 in account 1 . Note that this probability will be the only source of uncertainty in the differential paths we will consider (all the differential transitions through exclusive or operation always happen with probability equal to 1) and the product of all probabilities will be the core of the final complexity of the attack.

Also, one can check that the conditions on the Mill function input words are not necessarily independent. One may have to control differential transitions for nonlinear subfonctions located on adjacent positions (for example the first subfunction, involving $M_{0}$ and $M_{1}$, and the second, involving $M_{1}$ and $M_{2}$ ). This has two effects : potential incompatibility or condition compression (concerning $M_{1}$ in our example). In the first case, two conditions are located on the same input word and are contradicting (for example, one would have both $M_{1}=0^{w}$ and $M_{1}=1^{w}$ ). Thus, the differential path would be impossible to verify and, obviously, one has to avoid this scenario. For the second case, two conditions apply on the same input word but are not contradicting. Here, there is a chance that those conditions are redundant and we only have to account one time for a probability $2^{-w}$. Finally, note that all those aspects have to be handled during the differential path establishment and not during the search for a valid pair of messages.

\subsection{Control Words}

When trying to find a collision attack for a hash function, two major tools are used : the differential path and the freedom degrees. In the next section, we will describe how to find good differential paths using symmetric differences. If a given path has probability of success equal to $P$, the complexity of a naive attack would be $1 / P$ operations : if one chooses randomly and non-adaptively $1 / P$ random message input pairs that are coherent with the differential constraints, there is a rather good chance that a one of them will follow the differential path entirely. However, for the same differential path, the complexity of the attack can be significantly decreased if the attacker chooses its inputs in a clever and adaptive manner.

In the case of RadioGatún, $3 w$-bit message words are incorporated into the internal state at each round. Those words will naturally diffuse into the whole internal state, but not immediately. Thus, it is interesting to study how this diffusion behaves. Since the events we want to control through the differential path are the transitions of the nonlinear part of the Mill function (which depend on the input words of the Mill function), we will only study the diffusion regarding the input words of the Mill function.

Table 2 gives the dependencies between the message words incorporated at an iteration $k$, and the 19 input words of the Mill function at iteration $k, k+1$ and $k+2$. One can argue that a modification of a message block does not necessarily impacts the input word marked by a tick in Table 2 because the nonlinear

\footnotetext{
${ }^{1}$ In a dual view, all the conditions derived from Table 1 must be fulfilled.
} 
Table 2. Dependencies between the message words incorporated at an iteration $k$, and the 19 input words of the Mill function of RadioGatún at iteration $k, k+1$ and $k+2$. The first table (respectively second and third) gives the dependencies regarding the message block $m_{0}^{k}$ (respectively $m_{1}^{k}$ and $m_{2}^{k}$ ). The columns represent the input words of the Mill function considered and a tick denotes that a dependency exists between the corresponding input word and message block.

\begin{tabular}{|c||c|c|c|c|c|c|c|c|c|c|c|c|c|c|c|c|c|c|c|}
\hline iteration & $M_{0}$ & $M_{1}$ & $M_{2}$ & $M_{3}$ & $M_{4}$ & $M_{5}$ & $M_{6}$ & $M_{7}$ & $M_{8}$ & $M_{9}$ & $M_{10}$ & $M_{11}$ & $M_{12}$ & $M_{13}$ & $M_{14}$ & $M_{15}$ & $M_{16}$ & $M_{17}$ & $M_{18}$ \\
\hline \hline $\mathrm{k}$ & & & & & & & & & & & & & & & & & $\checkmark$ & & \\
\hline $\mathrm{k}+1$ & & $\checkmark$ & $\checkmark$ & & $\checkmark$ & $\checkmark$ & & & & $\checkmark$ & & & $\checkmark$ & $\checkmark$ & & & & $\checkmark$ & \\
\hline $\mathrm{k}+2$ & $\checkmark$ & $\checkmark$ & $\checkmark$ & $\checkmark$ & $\checkmark$ & $\checkmark$ & $\checkmark$ & $\checkmark$ & $\checkmark$ & $\checkmark$ & $\checkmark$ & $\checkmark$ & $\checkmark$ & $\checkmark$ & $\checkmark$ & $\checkmark$ & $\checkmark$ & $\checkmark$ & $\checkmark$ \\
\hline
\end{tabular}

\begin{tabular}{|c||c|c|c|c|c|c|c|c|c|c|c|c|c|c|c|c|c|c|c|}
\hline iteration & $M_{0}$ & $M_{1}$ & $M_{2}$ & $M_{3}$ & $M_{4}$ & $M_{5}$ & $M_{6}$ & $M_{7}$ & $M_{8}$ & $M_{9}$ & $M_{10}$ & $M_{11}$ & $M_{12}$ & $M_{13}$ & $M_{14}$ & $M_{15}$ & $M_{16}$ & $M_{17}$ & $M_{18}$ \\
\hline \hline $\mathrm{k}$ & & & & & & & & & & & & & & & & & & $\checkmark$ & \\
\hline $\mathrm{k}+1$ & & $\checkmark$ & & & $\checkmark$ & $\checkmark$ & & & & $\checkmark$ & & & $\checkmark$ & $\checkmark$ & & $\checkmark$ & $\checkmark$ & & \\
\hline $\mathrm{k}+2$ & $\checkmark$ & $\checkmark$ & $\checkmark$ & $\checkmark$ & $\checkmark$ & $\checkmark$ & $\checkmark$ & $\checkmark$ & $\checkmark$ & $\checkmark$ & $\checkmark$ & $\checkmark$ & $\checkmark$ & $\checkmark$ & $\checkmark$ & $\checkmark$ & $\checkmark$ & $\checkmark$ & $\checkmark$ \\
\hline
\end{tabular}

\begin{tabular}{|c||c|c|c|c|c|c|c|c|c|c|c|c|c|c|c|c|c|c|c|}
\hline iteration & $M_{0}$ & $M_{1}$ & $M_{2}$ & $M_{3}$ & $M_{4}$ & $M_{5}$ & $M_{6}$ & $M_{7}$ & $M_{8}$ & $M_{9}$ & $M_{10}$ & $M_{11}$ & $M_{12}$ & $M_{13}$ & $M_{14}$ & $M_{15}$ & $M_{16}$ & $M_{17}$ & $M_{18}$ \\
\hline \hline $\mathrm{k}$ & & & & & & & & & & & & & & & & & & & $\checkmark$ \\
\hline $\mathrm{k}+1$ & & $\checkmark$ & & & $\checkmark$ & $\checkmark$ & & $\checkmark$ & $\checkmark$ & & & & $\checkmark$ & & & $\checkmark$ & $\checkmark$ & & \\
\hline $\mathrm{k}+2$ & $\checkmark$ & $\checkmark$ & $\checkmark$ & $\checkmark$ & $\checkmark$ & $\checkmark$ & $\checkmark$ & $\checkmark$ & $\checkmark$ & $\checkmark$ & $\checkmark$ & $\checkmark$ & $\checkmark$ & $\checkmark$ & $\checkmark$ & $\checkmark$ & $\checkmark$ & $\checkmark$ & $\checkmark$ \\
\hline
\end{tabular}

function can sometimes "absorb" the diffusion of the modification. However, we emphasize that even if we depict here a behavior on average for the sake of clarity, all those details are taken in account thanks to our computer-aided use of the control words.

\section{An Improved Backtracking Search}

Our aim is to find internal collisions, i.e. collisions on the whole internal state before application of the blank rounds.

In order to build a good differential path using symmetric differences, we will use a computer-aided meet-in-the-middle approach, similar to the technique in [29]. More precisely, we will build our differential path $D P$ by connecting together separate paths $D P_{f}$ and $D P_{b}$. We emphasize that, in this section, we only want to build the differential path and not to look for a colliding pair of messages. $D P_{f}$ will be built in the forward direction starting from an internal state containing no difference (modeling the fact that we have no difference after the initialization of the hash function), while $D P_{b}$ will be built in the backward direction of the hash computation starting from an internal state containing no difference (modeling the fact that we want a collision at the end of the path).

Starting from an internal state with no difference, for each round the algorithm will go through all the possible difference incorporations of the message input (remember that we always use symmetric differences, thus we only have $2^{3}=8$ different cases to study) and all the possible symmetric differences transitions during the Mill function according to Table1 (the differential transitions through exclusive or operations are fully deterministic). The algorithm can be 
compared to a search tree in which the depth represents the number of rounds of RadioGatún considered and each node is a reachable differential internal state.

\subsection{Entropy}

An exhaustive search in this tree would obviously imply making useless computations (some parts of the tree provide too costly differential paths anyway). To avoid this, we always compute an estimation of the cost of finding a message pair fulfilling the differential paths during the building phase of the tree, from an initial state to the current leaf in the forward direction, and from the current leaf to colliding states in the backward direction.

A first idea would be to compute the current cost of $D P_{f}$ and $D P_{b}$ during the meet-in-the-middle phase. But, as mentioned in Section 3 some words of the mill only depend on the inserted message block after 1 or 2 rounds. Therefore, some conditions on the mill value have to be checked 2 rounds earlier, and some degrees of freedom may have to be used to fulfill conditions two rounds later. As $D P_{f}$ and $D P_{b}$ are computed round per round, it is difficult to compute their complexity during the search phase, while having an efficient early-abort algorithm.

Therefore, we use an ad hoc parameter, denoted $H^{k}$ and defined as follows. If $c^{k}$ is the total number of conditions on the mill input words at round $k$ (from Table 1), we have for a path of length $n$ :

$$
\left\{\begin{array}{l}
H^{k}=\max \left(H^{k+1}+c^{k}-3,0\right), \forall k<n \\
H^{n}=0
\end{array}\right.
$$

The idea is to evaluate the number of message pairs required at step $k$ in order to get $2^{w \times H^{k+1}}$ message pairs at step $k+1$ of the exhaustive search phase. To achieve this, one needs to fulfill $c^{k} \times w$ bit conditions on the mill input values, with $3 \times w$ degrees of freedom. Therefore, the values of $H^{k}$ can be viewed as the relative entropies on the successive values of the internal state during the hash computation.

The final collision search complexity would be $2^{w \times H_{\max }}$, where $H_{\max }$ is the maximum value of $H^{i}$ along the path, if the adversary could choose 3 words of his choice at each step, and if each output word of the Mill function depended on all the input words. In the case of RadioGatún, the computation cost is more complex to evaluate, and this is described in Section 5. The maximum entropy can be linked to the backtracking cost $C_{b}$, as defined in [4]. One has the relation $C_{b}=H_{\max }+3$. The difference between these two notions is that the backtracking cost takes in account the randomization of the input message pairs, which has a $\operatorname{cost} 2^{3 w}$.

\subsection{Differential Path Search Algorithm}

The path search algorithm works as follows. Keep in mind that the values of the entropy along the path are relative values - any constant value can therefore 
be added or subtracted to all the $H_{i}$. A zero entropy at step $i$ means that one expects $2^{0}=1$ message pair to follow the path until step $i$. To evaluate a path, we then set the minimal value of the entropy along the path to zero, the cost being the maximal value of the entropy. Therefore we first compute candidates for $D P_{f}$ with a modified breadth-first search algorithm, eliminating those for which the maximum entropy exceeds the minimum entropy by more than $8 \times w$ (because we want to remain much lower than the $9,5 \times w$ bound from the birthday paradox). The algorithm differs from a traditional breadth-first search as we do not store all the nodes, but only those with an acceptable entropy : to increase the probability of linking it to $D P_{b}$, one only stores the nodes whose entropy is at least $\left(H_{\max }-4\right) \times w$. We also store the state value of the previous node with entropy at least $\left(H_{\max }-4\right) \times w$, to enable an efficient backtracking process once the path is found.

We then compute $D P_{b}$, using a depth-first search among the backwards transitions of the Mill function, starting from colliding states. We set the initial entropy to $H^{n}=0$, and we do not search the states for which $H>8$ (same reason as for $D P_{f}$ : we want to remain much lower than the bound from the birthday paradox). For each node having an entropy at most 4 , we try to link it with a candidate for $D P_{f}$.

\subsection{Complexity of the Path Search Phase}

The total amount of possible values for a symmetric differential on the whole state is $2^{13 \times 3+19}=2^{58}$. We use the fact that for RadioGatún, the insertion of $M \oplus M^{\prime}$ can be seen as the successive insertions of $M$ and $M^{\prime}$ without applying the round function. Therefore, we can consider setting the words 16, 17, 18 of the stored mill to 0 by a message insertion before storing it in the forward phase, and doing the same in the backward phase before comparing it to forward values. Therefore, the space on which the meet-in-the-middle algorithm has to find a collision has approximately $2^{55}$ elements. We chose to store $2^{27}$ values of $D P_{f}$, and thus we have to compare approximately $2^{28}$ values for $D P_{b}$.

\section{The Collision Attack}

In this section, we depict the final collision attack, and compute its complexity. Once a differential path is settled, the derived collision attack is classic : we will use the control words to increase as much as possible the probability of success of the differential path.

\subsection{Description}

The input for this attack is a differential path, with a set of sufficient conditions on the values of the mill to ensure that a pair of messages follow the path. The adversary searches the colliding pairs in a tree, in which the nodes are messages following a prefix of the differential path. The leaves are messages following 
the whole differential path. Thanks to an early-abort approach, the adversary eliminates candidates as soon as they differ from the differential path. Nodes are associated with message pairs, or equivalently by the first message of a message pair - the second message is specified by the differential trail. Therefore, they will be denoted by the message they stand for. The sons of node $M$ are then messages $M \| b$, where $b$ is a given message block, and the hash computation of $M \| b$ fulfills all the conditions.

The adversary then uses a depth-first approach to find at least one node at depth $n$, where $n$ is the length of the differential path. It is based on the trail backtracking technique, described in [4, 29]. To decrease the complexity of the algorithm, we check the conditions on the words of the mill as soon as they cannot be modified anymore by a message word inserted later.

From Table 2, we know that the $k$-th included message block impacts some words of the mill before the $k$-th iteration of the Mill function, some other words before the $k+1$-th iteration, and the rest of the mill words before the $k+2$-th iteration. We recall that $m^{k}$ is the $k$-th inserted block, and we now set that $M_{j}^{k}$ is the value of the $j$-th mill word after the $k$-th message insertion. Let also $\hat{M}_{j}^{k}$ be the value of the $j$-th word of the mill after the $k$-th nonlinear function computation.

After inserting $m^{k}$, one can then compute $M_{16}^{k}, M_{17}^{k}, M_{18}^{k}$, but also $M_{j}^{k+1}$ for $j=\{1,2,4,5,7,8,9,12,13,15\}$, and $M_{j}^{k+2}$ for $j=\{0,3,6,10,11,14\}$.

Some other conditions imply differences or non-differences between state words, $M_{j}^{k} \oplus M_{j+1}^{k}$. When writing these variables as functions of the input message words at step $k$ and $k-1$, and of the state variables before message insertion $k-1$, one can notice the following : before the $k$-th message insertion, one can compute $M_{j}^{k} \oplus$ $M_{j+1}^{k}$, for $j=\{15,16,17,18\}, M_{j}^{k+2} \oplus M_{j+1}^{k+2}$ for $j=\{7,10\}$, and $M_{j}^{k+1} \oplus M_{j+1}^{k+1}$ for all other possible values of $j$. Therefore, the adversary has to check conditions on three consecutive values of the mill on message insertion number $k$.

The most naive way to do it would consist in choosing $m^{k}$ at random and hoping the conditions are verified, but one can use the following facts to decrease the number of messages to check:

- The conditions on words $M_{16}^{k}, M_{17}^{k}$ and $M_{18}^{k}$ as well as these on the values $M_{15}^{k} \oplus M_{16}^{k}, M_{16}^{k} \oplus M_{17}^{k}, M_{17}^{k} \oplus M_{18}^{k}$ and $M_{18}^{k} \oplus M_{0}^{k}$ at step $k$ can be fulfilled by $x o r$-ing the adequate message values at message insertion $k$.

- Using the linearity of all operations except the first one, the adversary can rewrite the values $M_{j}^{k+1}$ as a linear combination of variables $\hat{M}_{j}^{k}$, with $j=$ $\{0, \ldots, 18\}$. Words $\hat{M}_{0}^{k}$ to $\hat{M}_{13}^{k}$ do not depend on the last inserted message value, therefore can be computed before the message insertion.

- A system of equations in variables $\hat{M}_{14}^{k}, \ldots, \hat{M}_{18}^{k}$ remains. These equations are derived from conditions on round $k+1$, by reversing the linear part of the Mill function. More precisely, these equations define the possible values of these variables, or of the xor of two of these variables, one of them being rotated. 
The computation of the sons of a node at depth $k$ work as follows:

1. The adversary checks the consistency of the equations on $\hat{M}_{14}^{k}, \ldots, \hat{M}_{18}^{k}$. If these equations are not consistent, the adversary does not search the node. The probability that this system is consistent depends on dimension of the Kernel of the system and can be computed a priori.

2. The adversary exhausts the possible joint values of $\hat{M}_{14}^{k}, \ldots, \hat{M}_{18}^{k}, M_{16}^{k}, M_{17}^{k}$ and $M_{18}^{k}$, considering all the conditions on these variables, which can be expressed bitwise (as the nonlinear part of the Mill function also works bitwise). The cost of this phase is then linear in $w$. The mean number of sons depends on the number of conditions.

3. For each remaining message block, the adversary checks all the other linear conditions on $\hat{M}_{14}^{k}, \ldots, \hat{M}_{18}^{k}$ and the conditions on the mill values 2 rounds later.

\subsection{Computation of the Cost}

We will now explain how to compute the complexity of the collision search algorithm. The most expensive operation is the search of the sons of nodes. The total complexity of a given depth level $k$ is the product of the number of nodes that have to be explored at depth $k$ by the average cost of the search of these nodes. These parameters are exponential in $w$, therefore the total cost of the search can be approximated by the search of the most expensive nodes.

To compute the search cost, we assume that for all considered messages, the words of the resulting states for which no condition is imposed are independent and identically distributed. This is true at depth 0 , provided the attacker initializes the search phase with a long random message prefix. The identical distribution of the variables can be checked recursively, their independence is an hypothesis for the attack to work. This assumption is well-known in the field of hash function cryptanalysis for computing the cost associated to a differential path (see e.g. [29]).

Let $A^{k}$ be the number of nodes that have to be reached at depth $k$, and $C^{k}$ the average cost of searching one of these nodes. Let $P^{k}$ be the probability that a random son of a node at depth $k$ follows the differential path, and $Q^{k}$ the probability that a given node at depth $k$ has at least one valid son. At depth $k$, the average number of explored nodes is related to the average number of explored nodes at depth $k+1$. When only a few nodes are needed, the average case is not sufficient, and one has to evaluate the cost of finding at least one valid node of depth $k+1$.

One has the following relations, for $k \in\{0, \ldots, n-1\}$ :

$$
\left\{\begin{array}{l}
A^{k}=\max \left(\frac{A^{k+1}}{2^{3 w} P^{k}}, \frac{1}{Q^{k}}\right) \\
A^{n}=1
\end{array}\right.
$$

Let $K^{k}$ be the dimension of the Kernel of the linear system that has to be solved at depth $k$, and $\hat{P}^{k}$ the probability that the bitwise system of equations on the 
values of the mill before and after the nonlinear function has solutions. $\hat{P}^{k}$ can be computed exhaustively a priori for each value of $k$. A random node at depth $k$ has at least one valid son if the two following conditions happen :

- The bitwise conditions at depth $k$ and $k+1$ can be fulfilled,

- The remaining freedom degrees can be used to fulfill all the remaining conditions.

The first item takes in account the fact that some conditions might not depend on all the freedom degrees. Therefore, we have :

$$
Q^{k}=\min \left(2^{-K^{k}} \hat{P}^{k}, 2^{3 w-N_{C O N D}^{k}}\right)
$$

where $N_{C O N D}^{k}$ is the total number of conditions that has to be checked on the $k$-th message insertion. We also have $P^{k}=2^{-N_{C O N D}^{k}}$, because each condition is supposed to be fulfilled with probability half in the average case, which is true provided the free words - i.e. without conditions fixing their values, or linking it to another word - are i.i.d. .

Searching a node works as follows : one solves the bitwise system of equations on the values of $M_{16}, M_{17}, M_{18}, \hat{M}_{14}, \ldots, \hat{M}_{18}$. The set of message blocks that fulfill this equations system then has to be searched exhaustively to fulfill the other conditions, and to generate nodes at depth $k+1 . C^{k}$ is then the cost of this exhaustive search, and can be computed as the average number of message blocks that fulfill the system of equations. Therefore, we have $C^{k}=2^{3 w} \hat{P}^{k}$.

For each node at depth $k$, the attacker can first check the consistency of the conditions on the mill words at steps $k$ and $k+1$, which allows him not to search inconsistent nodes. Therefore, we have the following overall complexity:

$$
T=O\left(\max _{k}\left(\frac{C^{k} A^{k}}{2^{K^{k}}}\right)\right)
$$

The best path we found has complexity about $2^{11 \times w}$, which is above the security claimed by the designers of RadioGatún[4], it is given in Appendix. As a proof of concept, we also provide in Appendix an example of a colliding pair of messages following our differential path for RadioGatún with $w=2$. One can check that the observed complexity confirms the estimated one.

\subsection{Breaking the Birthday Bound}

Finding a final collision attack for RadioGatún with a computation complexity of $2^{11 w}$ required us to own a computer with a big amount of RAM for a few hours of computation. Yet, the memory and computation cost of the differential path search phase is determined by the $H_{\max }$ chosen by the attacker. We conducted tests that tend to show that the search tree is big enough in order to find a collision attack with an overall complexity lower than the birthday bound claimed by the designers 2 . The problem here is that the memory

${ }^{2}$ Note also that the size of the search tree can be increased by considering more complex symmetric differences, such as $0^{w}, 1^{w}, 01^{w / 2}$ and $10^{w / 2}$. 
and computation cost of the differential path search will be too big for nowadays computers, but much lower than the birthday bound. This explains why we are now incapable of providing a fully described collision attack for RadioGatún. However, we conjecture that applying our techniques with more memory and computation resources naturally leads to a collision attack for RadioGatún, breaking the ideal birthday bound.

\section{Conclusion}

In this paper, we presented an improved cryptanalysis of RadioGatún regarding collision search. Our attack can find collisions with a computation cost of about $2^{11 w}$ and negligible memory, which is by far the best known attack on this proposal.

We also gave arguments that shows that RadioGatún might not be a collision resistant hash function. We conjecture that applying our differential path search technique with more constraints will lead to collision attacks on RadioGatún.

\section{Acknowledgments}

The authors would like to thank Guido Bertoni, Joan Daemen, Michal Peeters annd Gilles Van Assche for their comments on sponge functions.

\section{References}

1. Andreeva, E., Neven, G., Preneel, B., Shrimpton, T.: Seven-Property-Preserving Iterated Hashing: ROX. In: Kurosawa, K. (ed.) ASIACRYPT 2007. LNCS, vol. 4833, pp. 130-146. Springer, Heidelberg (2007)

2. Bellare, M., Ristenpart, T.: Multi-Property-Preserving Hash Domain Extension and the EMD Transform. In: Lai, X., Chen, K. (eds.) ASIACRYPT 2006. LNCS, vol. 4284, pp. 299-314. Springer, Heidelberg (2006)

3. Bertoni, G., Daemen, J., Peeters, M., Van Assche, G.: Keccak specifications. Submission to NIST (2008)

4. Bertoni, G., Daemen, J., Peeters, M., Van Assche, G.: Radiogatun, a belt-and-mill hash function. Presented at Second Cryptographic Hash Workshop, Santa Barbara, August 24-25 (2006), http://radiogatun.noekeon.org/

5. Bertoni, G., Daemen, J., Peeters, M., Van Assche, G.: On the Indifferentiability of the Sponge Construction. In: Smart, N.P. (ed.) EUROCRYPT 2008. LNCS, vol. 4965, pp. 181-197. Springer, Heidelberg (2008)

6. Bertoni, G., Daemen, J., Peeters, M., Van Assche, G.: Sponge Functions. Presented at ECRYPT Hash Workshop (2007)

7. Biham, E., Dunkelman, O.: A framework for iterative hash functions: Haifa. In: Second NIST Cryptographic Hash Workshop (2006)

8. Bouillaguet, C., Fouque, P.-A.: Analysis of radiogatun using algebraic techniques. In: Keliher, L., Avanzi, R., Sica, F. (eds.) SAC 2008. LNCS. Springer, Heidelberg (2008) 
9. Brassard, G. (ed.): CRYPTO 1989. LNCS, vol. 435. Springer, Heidelberg (1990)

10. Bresson, E., Canteaut, A., Chevallier-Mames, B., Clavier, C., Fuhr, T., Gouget, A., Icart, T., Misarsky, J.-F., Naya-Plasencia, M., Paillier, P., Pornin, T., Reinhard, J.-R., Thuillet, C., Videau, M.: Shabal - a submission to advanced hash standard. Submission to NIST (2008)

11. Claesen, L.J.M., Daemen, J., Genoe, M., Peeters, G.: Subterranean: A $600 \mathrm{mbit} / \mathrm{sec}$ cryptographic vlsi chip. In: ICCD, pp. 610-613 (1993)

12. Cramer, R. (ed.): EUROCRYPT 2005. LNCS, vol. 3494. Springer, Heidelberg (2005)

13. Daemen, J.: Cipher and hash function design strategies based on linear and differential cryptanalysis. PhD thesis, Katholieke Universiteit Leuven (1995)

14. Daemen, J., Clapp, C.S.K.: Fast hashing and stream encryption with panama. In: Vaudenay, S. (ed.) FSE 1998. LNCS, vol. 1372, pp. 60-74. Springer, Heidelberg (1998)

15. Damgård, I.: A Design Principle for Hash Functions. In: Brassard [9], pp. 416-427

16. Dean, R.D.: Formal aspects of mobile code security. PhD thesis. Princeton University, Princeton (1999)

17. Fuhr, T., Peyrin, T.: Cryptanalysis of Radiogatún (2008)

18. Gorski, M., Lucks, S., Peyrin, T.: Slide attacks on hash functions. In: Pieprzyk, J. (ed.) ASIACRYPT 2008. LNCS, vol. 5350, pp. 143-160. Springer, Heidelberg (2008)

19. Joux, A.: Multicollisions in Iterated Hash Functions. Application to Cascaded Constructions. In: Franklin, M.K. (ed.) CRYPTO 2004. LNCS, vol. 3152, pp. 306-316. Springer, Heidelberg (2004)

20. Kelsey, J., Kohno, T.: Herding Hash Functions and the Nostradamus Attack. In: Vaudenay, S. (ed.) EUROCRYPT 2006. LNCS, vol. 4004, pp. 183-200. Springer, Heidelberg (2006)

21. Kelsey, J., Schneier, B.: Second Preimages on n-Bit Hash Functions for Much Less than $2^{\mathrm{n}}$ Work. In: Cramer [12], pp. 474-490

22. Khovratovich, D.: Two attacks on radiogatun. In: Chowdhury, D.R., Rijmen, V., Das, A. (eds.) INDOCRYPT 2008. LNCS, vol. 5365, pp. 53-66. Springer, Heidelberg (2008)

23. Khovratovich, D.: Cryptanalysis of hash functions with structures. Presented at ECRYPT Hash Workshop (2008)

24. Knudsen, L.R., Rechberger, C., Thomsen, S.S.: The Grindahl Hash Functions. In: Biryukov, A. (ed.) FSE 2007. LNCS, vol. 4593, pp. 39-57. Springer, Heidelberg (2007)

25. Lucks, S.: A Failure-Friendly Design Principle for Hash Functions. In: Roy, B.K. (ed.) ASIACRYPT 2005. LNCS, vol. 3788, pp. 474-494. Springer, Heidelberg (2005)

26. Maurer, U.M., Tessaro, S.: Domain Extension of Public Random Functions: Beyond the Birthday Barrier. In: Menezes, A. (ed.) CRYPTO 2007. LNCS, vol. 4622, pp. 187-204. Springer, Heidelberg (2007)

27. Menezes, A.J., Vanstone, S.A., Van Oorschot, P.C.: Handbook of applied cryptography. CRC Press, Inc., Boca Raton (1996)

28. Merkle, R.C.: One Way Hash Functions and DES. In: Brassard [9], pp. 428-446

29. Peyrin, T.: Cryptanalysis of Grindahl. In: Kurosawa, K. (ed.) ASIACRYPT 2007. LNCS, vol. 4833, pp. 551-567. Springer, Heidelberg (2007)

30. Rijmen, V., Van Rompay, B., Preneel, B., Vandewalle, J.: Producing collisions for panama. In: Matsui, M. (ed.) FSE 2001. LNCS, vol. 2355, pp. 37-51. Springer, Heidelberg (2001) 
31. Shoup, V. (ed.): CRYPTO 2005. LNCS, vol. 3621. Springer, Heidelberg (2005)

32. Wang, X., Lai, X., Feng, D., Chen, H., Yu, X.: Cryptanalysis of the hash functions md4 and ripemd. In: Cramer [12], pp. 1-18

33. Wang, X., Yin, Y.L., Yu, H.: Finding collisions in the full sha-1. In: Shoup [31], pp. $17-36$

34. Wang, X., Yu, H.: How to break md5 and other hash functions. In: Cramer [12], pp. $19-35$

35. Wang, X., Yu, H., Yin, Y.L.: Efficient collision search attacks on sha-0. In: Shoup [31], pp. $1-16$

\section{Appendix A: Collision for RadioGatún $[2]$}

To generate a collision for RadioGatún[2], we use a 143-block differential path of cost $2^{11 w}$.

We give here a collision for the 2-bit version of RadioGatún. One can easily check that it follows the differential path given above. We write the message words using values between 0 and 3, which stand for the possible values of 2-bit words. The differential path, and some statistics about the collision search, can be found in the longer version of this paper [17].

To ensure that one has enough starting points, we used a 5-block common prefix.

The two colliding messages are :

$$
\begin{aligned}
& M_{0}=330000000000000113311012012112300202 \\
& 020302233030030000223222220111000010 \\
& \begin{array}{llllllllllll}
031 & 001 & 033 & 020 & 000 & 000 & 222 & 103 & 110 & 312 & 231 & 321
\end{array}
\end{aligned}
$$

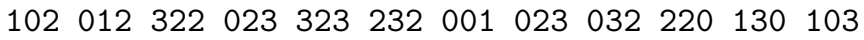

$$
\begin{aligned}
& 203003200232023011222222133110211031
\end{aligned}
$$

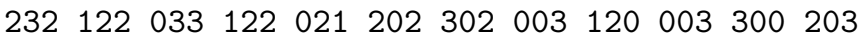

$$
\begin{aligned}
& \begin{array}{llllllllllll}
133 & 021 & 302 & 311 & 101 & 031 & 200 & 003 & 013 & 231 & 032 & 312
\end{array} \\
& 002202131331122201333301032230031220 \\
& 012130312100020322222220201012000201 \\
& 200 \quad 010230130310330201103130210102001 \\
& 200321112110232223010301213000133123
\end{aligned}
$$

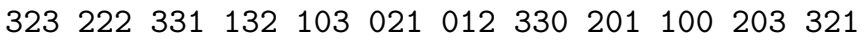

$$
\begin{aligned}
& 013332020000
\end{aligned}
$$




\section{$M_{1}=330000000000000113 \quad 311312022 \quad 122030202$ 020332103303303003113222120121030020 $\begin{array}{llllllllllll}031 & 001 & 303 & 313 & 000 & 330 & 222 & 103 & 110 & 312 & 202 & 321\end{array}$ $\begin{array}{llllllllllll}201 & 011 & 022 & 010 & 313 & 202 & 031 & 023 & 032 & 120 & 130 & 103\end{array}$ $\begin{array}{llllllllllll}200 & 303 & 233 & 232 & 013 & 321 & 111 & 211 & 203 & 123 & 121 & 031\end{array}$ 132112300122011202032003210300300100 203311302012101002100303013231302322 $\begin{array}{llllllllllll}032 & 131 & 102 & 001 & 211 & 232 & 300 & 301 & 302 & 230 & 301 & 120\end{array}$ $011103022200013022212113131311003 \quad 131$ $\begin{array}{llllllllllll}200 & 010 & 230 & 200 & 020 & 000 & 231 & 103 & 100 & 113 & 132 & 031\end{array}$ $\begin{array}{llllllllllll}233 & 321 & 112 & 220 & 232 & 220 & 010 & 332 & 223 & 300 & 100 & 123\end{array}$ $\begin{array}{llllllllllll}013 & 122 & 302 & 131 & 200 & 311 & 012 & 300 & 202 & 230 & 133 & 321\end{array}$ 013331023003}

The common value of the internal state is then :

$$
\begin{array}{r}
\operatorname{belt}[0]=(0,0,2,1,2,0,3,0,2,1,1,1,3), \\
\operatorname{belt}[1]=(3,1,0,2,3,2,2,3,1,2,3,0,2), \\
\operatorname{belt}[2]=(2,3,3,2,2,2,1,1,1,3,2,0,3), \\
\operatorname{mill}=(2,0,2,2,1,0,1,0,3,1,3,3,2,2,3,3,0,3,3)
\end{array}
$$

